\title{
GMR
}

\section{Molecular characterization of the gene checkpoint homolog 1 in Daphnia carinata during different reproductive phases}

\author{
L. Kong ${ }^{1 *}$, H.X. $\mathrm{Li}^{1 *}$, D.L. Wu ${ }^{1}$, G.R. Xu' ${ }^{1}$, D.L. Wang ${ }^{2}$ and Y.L. Zhao ${ }^{1}$ \\ ${ }^{1}$ School of Life Science, East China Normal University, Shanghai, China \\ ${ }^{2}$ School of Marine Sciences, Ningbo University, Ningbo, China \\ *These authors contributed equally to this study. \\ Corresponding author: Y.L. Zhao \\ E-mail: ylzhao@bio.ecnu.edu.cn
}

Genet. Mol. Res. 15 (2): gmr.15026132

Received November 22, 2015

Accepted January 22, 2016

Published April 4, 2016

DOI http://dx.doi.org/10.4238/gmr.15026132

\begin{abstract}
Full-length cDNA of the gene checkpoint homolog 1 (Chk1) was cloned from Daphnia carinata and designated DcarChk1. DcarChk1 cDNA was 1817 bp in length and encoded a 497-amino acid polypeptide. Phylogenetic analyses revealed that DcarChk1 was most closely related to Chk1 of Daphnia pulex, followed by homologous genes of insects. Expression of DcarChk1 was higher in adult Daphnia than in larvae, and significantly higher in males than females, as determined by real-time polymerase chain reaction analysis. Using whole-mount in situ hybridization techniques, DcarChk1 in parthenogenetic females was found to be expressed mainly on the head surface, capillus, and carapace valve edge. In contrast, in sexual females, DcarChk1 was expressed mainly in the joint of the second antenna, and in the thoracic limbs and capillus. These results suggest that DcarChk1 plays a significant role in both the growth and development, as well as in regulating reproductive plasticity, in $D$. carinata.
\end{abstract}

Key words: Daphnia carinata; Cloning; Expression; Whole-mount in situ hybridization; Checkpoint homolog 1 


\section{INTRODUCTION}

Checkpoint homolog 1 (Chk1) is a type of serine/threonine-protein kinase gene, and is involved in regulating the cell cycle checkpoint by DNA damage (Merry et al., 2010). It was initially discovered and identified as a cell cycle detection gene in fission yeast (Walworth et al., 1993). Subsequently, homologs have been found in mammals, insects, birds, amphibians, budding yeast, and other eukaryotes; all have a highly conserved structure and function (Chen and Sanchez, 2004). Chk1 is the downstream target of the ataxia telangiectasia-mutated (ATR) and ataxia telangiectasia-mutated gene (ATM) (Liu et al., 2010), which are sensors in the signal transduction pathway of the DNA damage checkpoint (Li et al., 2006). When DNA is damaged, ATR and ATM are activated and regulate different signal factors to end the cell cycle in G2/M (Wang et al., 2006a; Pan et al., 2009). Chk1 is the core molecular check for damaged DNA in G2/M. As a transducin, it can transmit amplifying signal to the effector Cdc25c, which enacts phosphorylation of Cdc25c to repair damaged DNA (Rouse and Jackson, 2002; Ling et al., 2010). Hence, when expression levels of Chk1 decrease, damaged DNA is no longer detected or repaired efficiently, which leads to cell apoptosis (Sørensen et al., 2005; Syljuåsen et al., 2005; Bolderson et al., 2009; Carrassa et al., 2009). Clinical studies have found that Chk1 is expressed in a variety of tumor cells (Bartek and Lukas, 2003). Moreover, other studies have shown that RNA interference silences Chk1 and reduces its expression, resulting in limited reparation of tumor cells and increased mortality. A robust understanding of the expression levels of Chk1 will, therefore, be particularly useful for the advancement of cancer therapies (Slupianek et al., 2002; Zhang et al., 2008; Chen et al., 2009).

Daphnia carinata is a common microzooplankton which, because of its high protein content, is used as a high quality feed in commercial fisheries (Yang, 1994). It is a small organism, is fast breeding and readily cultivated, and is sensitive to environmental variation; these characteristics make it ideal as a biological indicator of water quality in environmental monitoring studies (Qi et al., 1991). Compared to other invertebrates, cladocerans such as Daphnia spp have a unique life history that involves facultative parthenogenesis. Under suitable conditions, Daphnia spp reproduce parthenogenetically, but as conditions deteriorate, bisexual reproduction occurs. Eggs produced withstand the harsh environment, and hatch when conditions improve (Hebert, 1978). This reproductive plasticity is an adaptive advantage in variable environments, and ensures population persistence (Stross and Hill, 1965). While the ecology of this reproductive plasticity has received much attention (Martinez-Jeronimo et al., 1994; Khmeleva et al., 1995; Cao et al., 2001), knowledge of regulatory molecular mechanisms is lacking. In this study, we use molecular tools to explore the potential role of Chk1 in regulating growth and reproduction in D. carinata. The data presented on the expression pattern and function of DcarChk1 in D. carinata provide a basis for understanding the molecular mechanisms driving growth and reproductive plasticity in cladocerans.

\section{MATERIAL AND METHODS}

\section{Sample preparation}

D. carinata were obtained from the College of Life Sciences of Ningbo University. Healthy parthenogenetic individuals were light cultured at $25^{\circ} \mathrm{C}$ and fed with cladoceran nutrient solution for 2 to 3 weeks. The solution contained $1.5 \mathrm{~g}$ cow dung, $2 \mathrm{~g}$ vegetables, $20 \mathrm{~g}$ fertile soil, and $1 \mathrm{~L}$ water. The vegetables were shredded and boiled for 5 to $6 \mathrm{~min}$, allowed to cool to $60^{\circ}$ to $70^{\circ} \mathrm{C}$, and then 
the dung and soil were added and the solution filtered. Parthenogenetic and sexual females $(\mathrm{N}=$ 150 ) were isolated, frozen in liquid nitrogen, and stored at $-80^{\circ} \mathrm{C}$ for RNA extraction. A further 30 individuals were fixed in $4 \%$ paraformaldehyde at $4^{\circ} \mathrm{C}$ overnight, after which the paraformaldehyde was replaced with $100 \%$ methanol, and specimens were stored at $-20^{\circ} \mathrm{C}$ for whole-mount in situ hybridization.

\section{Primer design}

Forward (F1) and reverse (R1) primers were designed to amplify the conserved region of DcarChk1 from sequence of the Chk1 homolog of Daphnia pulex (GenBank accession No. AGN95867; Table 1). Based on this partial sequence, two pairs of gene-rapid amplification of cDNA ends (RACE) primers (F2, R2; Table 1) were designed for RACE-polymerase chain reaction (PCR) in order to clone the whole gene sequence. Full-length DcarChk1 was used to design a pair of primers for quantitative PCR (qPCR), F3, and R3, targeted to the open reading frame (ORF) sequences of the gene (Table 1). Specific primers to amplify the L8 ribosomal gene (L8-F, L8-R; Table 1) were designed according to the conserved sequence. For whole-mount in situ hybridization, upstream (F4) and downstream (R4) primers in the ORF of DcarChk1 were designed for the preparation of riboprobe (Table 1). All primers were purchased commercially (Sangon, China).

\begin{tabular}{|c|c|c|}
\hline Primer & Sequence $\left(5^{\prime}-3^{\prime}\right)$ & Purpose \\
\hline F1 & TGGGTGAAGGAGCTTTTGGAGAGG & \multirow[t]{2}{*}{ Standard PCR } \\
\hline R1 & ATCCCAAGGCAATTCTCCGGC & \\
\hline F2 & CCAATGCTGCTGAAACGGTCAAGA & \multirow[t]{2}{*}{ RACE-PCR } \\
\hline R2 & GGGGCATTCCCACATCAGGCTCTAT & \\
\hline \multirow[t]{3}{*}{ UPM } & CTAATACGACTCACTATAGGGCAAGC & \multirow[t]{3}{*}{ Kit primers } \\
\hline & AGTGGTATCAACGCAGAGT & \\
\hline & CTAATACGACTCACTATAGGGC & \\
\hline F3 & AGGCGCAGTGTTTAGCTTCT & \multirow[t]{2}{*}{ Real-Time PCR } \\
\hline R3 & CCGGGGGTGTGTATTTTCCA & \\
\hline L8-F & CCGAGGTCCTATTCCATCAT & \multirow[t]{2}{*}{ L8 reference gene } \\
\hline L8-R & TCGGATGTCTGTCTTCGGC & \\
\hline F4 & TTGGGTTTCTTCTCCGGTTC & \multirow[t]{2}{*}{ Riboprobe } \\
\hline R4 & TGGCCATTGACCATGTTACT & \\
\hline
\end{tabular}

\section{RNA extraction and first-strand cDNA synthesis}

Total RNA was extracted using an RNA extraction kit (Axygen, USA) according to the manufacturer protocol. The degree of RNA degradation was determined by agarose gel electrophoresis. The concentration and optical density (OD) values of samples were measured using a nucleic acid protein detector. Clear bands with no tail and OD values between 1.8 and 2.0 $\mathrm{ng} / \mu \mathrm{L}$ indicated that RNA extraction was successful. For standard and qPCR, first-strand cDNA was synthesized using a reverse transcription kit (TaKaRa, Japan). For RACE-PCR, a SMART ${ }^{T M}$ RACE cDNAAmplification Kit (Clontech, USA) was used. Five prime- and 3'-RACE-ready cDNA templates were prepared according to the manufacturer instructions. Synthesized DNA was stored at $-20^{\circ} \mathrm{C}$.

\section{Cloning of partial- and full-length DcarChk1}

Using first-strand cDNA of adult parthenogenetic Daphnia as a template and a Premix Ex Taq $^{\text {TM }}$ HS Kit (TaKaRa, Japan), DcarChk1 partial sequence was generated. Amplification by PCR 
was performed in a total volume of $25 \mu \mathrm{L}$ under the following conditions: $94^{\circ} \mathrm{C}$ for $5 \mathrm{~min}$; 35 cycles of $94^{\circ} \mathrm{C}$ for $30 \mathrm{~s}, 60^{\circ} \mathrm{C} 30 \mathrm{~s}$, and $72^{\circ} \mathrm{C}$ for $1 \mathrm{~min}$; and a final extension of $72^{\circ} \mathrm{C}$ for $10 \mathrm{~min}$, followed by storage at $4^{\circ} \mathrm{C}$. The PCR products were separated using $1.2 \%$ agarose $(\mathrm{w} / \mathrm{v})$ gel electrophoresis, and the appropriate bands were sent to Major Biotechnology Co., Ltd (Shanghai) for sequencing. BLAST was used to compare products against the NCBI database; if similarity was greater than $80 \%$ and gene names were consistent, then partial cDNA sequence of DcarChk1 was confirmed. Full-length cDNA of DcarChk1 was generated by further RACE-PCR amplification, under the same PCR conditions described above. The melting temperatures of 5'- and 3'-RACE primers were $58^{\circ}$ and $63^{\circ} \mathrm{C}$, respectively. Amplification products were separated using agarose gel electrophoresis and the appropriate bands were cloned and sequenced by Major Biotechnology Co., Ltd.

\section{Bioinformatics analysis of DcarChk1}

Sequencing results for the 3'- and 5'-RACE PCR products were screened against the NCBI database, splicing the right 5' and 3' sequences to confirm the full-length cDNA of DcarChk1. For comparative analyses of gene and protein sequences, the NCBI BlastX tool (http://www.ncbi. nlm.nih.gov) was used. For analysis of ORFs and predicted amino acid (aa) sequences, the NCBI ORF Finder (http://www.ncbi.nlm.nih.gov/gorf/orfig.cgi) was used. The isoelectric point (pl) and molecular weight (MW) of proteins were calculated using the EXPASY database (http://web.expasy. org/cgi-bin/protscale/protscale.pl). The SignalP software (http://www.cbs.dtu.dk/services/signalp) was used for signal peptide identification. Characteristics of the structural domain were analyzed using the Simple Modular Architecture Research Tool SMART (http://www.smart.emblheidelberg. de). The BLAST feature of the NCBI database was used to extract protein names, sequences, and aa numbers for the phylogenetic analysis. ClustalW2 (http://www.ebi.ac.uk/tools/msa/clustalw2) was used for multiple sequence alignment of Chk1 genes, and the MEGA 4.0 software was used to construct the phylogenetic tree using the neighbor-joining method with a bootstrap value of 1000 .

\section{Real-time qPCR analysis}

The concentration of cDNA templates in different growth and reproductive phases, and qPCR target gene expression was normalized against that of the L8 ribosomal gene. Reactions contained $12.5 \mu \mathrm{L}$ SYBR Premix Ex Taq II, $1 \mu \mathrm{L}$ cDNA template, $0.5 \mu \mathrm{L}$ primer F3 (10 $\mu \mathrm{M}), 0.5 \mu \mathrm{L}$ primer R3 $(10 \mu \mathrm{M})$, and $\mathrm{ddH}_{2} \mathrm{O}$ to form a final volume of $25 \mu \mathrm{L}$. Conditions for PCR were $95^{\circ} \mathrm{C}$ for $2 \mathrm{~min}$, then 40 cycles of $95^{\circ} \mathrm{C}$ for $15 \mathrm{~s}$ and $60^{\circ} \mathrm{C}$ for $30 \mathrm{~s}$. Samples were assayed in triplicate, and melting and amplification curves were constructed from the results. Gene expression was calculated using the $2^{-\Delta \Delta C t}$ method (Livak and Schmittgen, 2001). One-way ANOVA was used to assess significance differences in DcarChk1 expression among different reproductive phases; analyses were undertaken using the SPSS software.

\section{Riboprobe preparation}

Using the upstream (F4) and downstream (R4) primers described above (Table 1), PCR amplification was used to prepare in situ hybridization probes, with a probe length of $499 \mathrm{bp}$, and a melting temperature of $57^{\circ} \mathrm{C}$. Products were separated by electrophoresis on $1.2 \%$ agarose (w/v) gel extracted using a QIAquick Gel Extraction kit. Purified products were ligated into the 
pGEM-T vector (Promega, USA) using T4 DNA ligase (Promega, USA) and then transformed into Escherichia coli strain DH5 $\alpha$. Following blue-white screening, positive clone bacterium liquid was sent to BioSune Biotechnology Co. Ltd. (Shanghai) for sequencing. Bacterium solutions positive for target inserts were cultured and the plasmid extracted. According to the multiple cloning site of PGEM-T Vector and the restriction enzyme cutting site of the DcarChk1 gene probe sequence, the restriction enzymes $\mathrm{Ncol}$ and Spel were used for linearization. Digested products were gel-purified and used as sense and antisense probes of DcarChk1 template. Transcription systems $(20 \mu \mathrm{L})$ were set up in 1.5-mL centrifuge tubes containing: $4 \mu \mathrm{L} 5 \mathrm{X}$ transcription buffer; $2 \mu \mathrm{L}$ DTT (100 mM); $0.5 \mu \mathrm{L}$ RNase inhibitor (40 U); $8 \mu \mathrm{L}$ linear DNA (<1 $\mu \mathrm{g}) ; 2 \mu \mathrm{L}$ 10X DIG labeling mixture; $1 \mu \mathrm{L}$ SP6 or T7 RNA polymerase $(20 \mathrm{U} / \mu \mathrm{L})$; and $2.5 \mu \mathrm{L} 0.1 \%$ diethylpyrocarbonate (DEPC) Millipore $\mathrm{H}_{2} \mathrm{O}$. Reactions were mixed and incubated at $37^{\circ} \mathrm{C}$ for $2 \mathrm{~h}$. Then, $1 \mu \mathrm{L}$ DNase (RNase-free) was added and incubation was continued for $15 \mathrm{~min}$. A $1 / 9$ volume of $5 \mathrm{M} \mathrm{LiCl}$ and 2 volumes of absolute ethanol were added and incubation continued at $-20^{\circ} \mathrm{C}$ overnight. Mixtures were centrifuged at $12,000 \mathrm{rpm}$ for $30 \mathrm{~min}$ at $4^{\circ} \mathrm{C}$ and supernatants discarded. Then, RNA pellets were washed twice with $75 \%$ ethanol, centrifuged at $13,000 \mathrm{rpm}$ for $5 \mathrm{~min}$, and thoroughly dried to remove residual ethanol. Lastly, RNA sediment was re-suspended in $30 \mu \mathrm{L} \mathrm{DEPC} \mathrm{water,} \mathrm{and} 1.5 \mu \mathrm{L}$ RNA inhibitor (20 $\mathrm{U})$ was added. Aliquots of RNA solutions $(1 \mu \mathrm{L})$ were added and electrophoresed; concentrations were measured while remaining RNA probes were stored at $-20^{\circ} \mathrm{C}$.

\section{Whole-mount in situ hybridization}

Whole-mount in situ hybridization was carried out according to previously published methods (Thisse and Thisse, 2008; Zhang and Liu, 2013) with some modifications. Briefly, on the first day, specimens stored at $-20^{\circ} \mathrm{C}$ were rehydrated gradually with methanol-PBST and digested with proteinase $\mathrm{K}(10 \mu \mathrm{g} / \mathrm{mL})$. Sexually reproducing and parthenogenetic individuals were digested at $37^{\circ} \mathrm{C}$ for 15 and $12 \mathrm{~min}$, respectively. Following pre-hybridization at $67^{\circ} \mathrm{C}$ for $2.5 \mathrm{~h}$, $100 \mu \mathrm{L}$ RNA probe diluted 1:100 was added and incubation continued at $67^{\circ} \mathrm{C}$ overnight. On the second day, probes were aspirated and residuum washed gradually with hybridization 2 X SSC and $0.2 X$ SSC-MABT. Specimens were blocked for approximately $2 \mathrm{~h}$ at room temperature with slow shaking in MAB block solution. Then, anti-DIG antibody (diluted 1:5000; Roche, USA) was added and specimens incubated at $4^{\circ} \mathrm{C}$ for $13 \mathrm{~h}$. On the third day, antibody solution was discarded and specimens were washed briefly in MABT. Then, NBT liquid dye (Roche) and TSM2 were added at a ratio of 1:50 and specimens incubated at room temperature in the dark for approximately $10 \mathrm{~min}$. Finally, individuals were fixed in $4 \%$ paraformaldehyde. Hybridization was visualized and recorded using a BX51 fluorescence microscope (Olympus, USA).

\section{RESULTS}

\section{Cloning of full-length DcarChk1 cDNA}

Using $D$. carinata template cDNA and gene-specific primers, a 584-bp fragment was obtained by PCR amplification. BLASTx comparison showed high homology to the gene homolog of $D$. pulex from the NCBI database. On this basis, gene-RACE primers were designed, and 5'and 3'-RACE PCR yielded 1442- and 631-bp fragments, respectively. Fragments were spliced with an intermediate fragment in order to construct full-length (1817 bp) DcarChk1 cDNA (GenBank accession No. KJ735446; Figure 1). 
1 atggggacatttgagtcaaaatgccacctgttctggtaatgaaatcaattgtgtgat

58 ttctgctacgtgagttcaatccgtgggtgactattcatttaacggtgttaaaaagccacc

118 gaaaagacgcggaaaaagtggtaatacgtgttagcttgtcgtcttttatctgtgtcttaa

178 cgggttgtcaccttcgcacctccttgtgatctgttgtaaagaaagtatttatcaccaatt

238 atggatgaaaaaagtaaaaagtatgaaggaaaaaacagtgtaattgaatttgtagaaggt

$\begin{array}{lllllllllllllllllllll}1 & M & D & E & K & S & K & K & Y & E & G & K & N & S & V & I & E & F & V & E & G\end{array}$

298 tgggatatgatacagacactgggtgaaggagcgtttggggaggtaaaactactagtcaat

$\begin{array}{lllllllllllllllllllll}21 & \text { W } & \text { D } & \text { M } & \text { I } & \text { Q } & \text { T } & \text { L } & G & \text { E } & G & \text { A } & \text { F } & \text { G } & \text { E } & \text { V } & \text { K } & \text { L } & \text { L } & \text { V } & \text { N }\end{array}$

358 gcaaaaactggagaagcagtggcaatgaaagtaattgatttgaagaaacatgccaatgct

$\begin{array}{lllllllllllllllllllll}41 & \text { A } & \text { K } & T & G & E & A & V & A & M & K & V & \text { I } & \text { D } & \text { L } & \text { K } & \text { K } & \text { H } & \text { A } & \text { N } & \text { A }\end{array}$

418 gctgaaacggtcaagaaagaagtttgtgttcacagaatgttaaatgaccctcatgtcatt

$\begin{array}{rllllllllllllllllllll}61 & A & E & T & V & K & K & E & V & C & V & H & R & M & L & N & D & P & H & V & \text { I }\end{array}$

478 cggttttatggcagaagggaaaatggcaattttgaattcattttttggaatatgcaagt

$\begin{array}{lllllllllllllllllllll}81 & R & F & Y & G & R & R & E & N & G & N & F & E & F & I & F & L & E & Y & A & S\end{array}$

538 ggtggagagctctttgacagaatagagcctgatgtgggaatgccccaaatggaagcccag

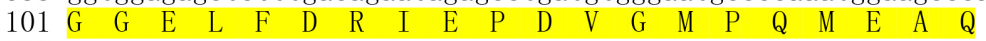

598 cgttattcaaacaattgattgctggagtaaattacttgcatagcagaggagttgctcat

$\begin{array}{llllllllllllllllllllll}121 & R & Y & F & K & Q & \text { L } & \text { I } & \text { A } & \text { G } & \text { V } & \text { N } & \text { Y } & \text { L } & \text { H } & \text { S } & \text { R } & \text { G } & \text { V } & \text { A } & \text { H }\end{array}$

658 agagacattaaaccagaaaatttgcttttggatgcaaatgataatt gaaaatatctgat

$\begin{array}{lllllllllllllllllllll}141 & \mathrm{R} & \mathrm{D} & \mathrm{I} & \mathrm{K} & \mathrm{P} & \mathrm{E} & \mathrm{N} & \mathrm{L} & \mathrm{L} & \mathrm{L} & \mathrm{D} & \mathrm{A} & \mathrm{N} & \mathrm{D} & \mathrm{N} & \mathrm{L} & \mathrm{K} & \mathrm{I} & \mathrm{S} & \mathrm{D}\end{array}$

718 tttggaatggcgacaattttcgatttcaaggtcgagaaagacttctggataaacgttgt

$\begin{array}{lllllllllllllllllllll}161 & F & G & M & A & T & I & F & R & F & Q & G & R & E & R & L & L & D & K & R & C\end{array}$

778 ggaactttgccttacatagctcctgaagtgctttgtcgcaagtacgcagcagaaccggct

$\begin{array}{lllllllllllllllllllll}181 & \text { G } & \text { T } & \text { L } & \text { P } & \text { Y } & \text { I } & \text { A } & \text { P } & \text { E } & \text { V } & \text { L } & \text { C } & \text { R } & \text { K } & \text { Y } & \text { A } & \text { A } & \text { E } & \text { P } & \text { A }\end{array}$

838 gatatttggtcttgtggcgtcgtccttgttgccatgttagctggagaattaccttgggat

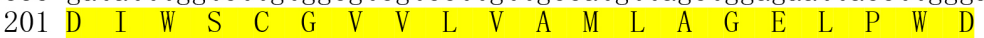

898 gtcccttctaatgattgtcctctctatacatcatggaaagaatgtcagataacgcgattg

$\begin{array}{lllllllllllllllllllll}221 & \text { V } & \text { P } & \text { S } & \text { N } & \text { D } & \text { C } & \text { P } & \text { L } & \text { Y } & \text { T } & \text { S } & \text { W } & \text { K } & \text { E } & \text { C } & \text { Q } & \text { I } & \text { T } & \text { R } & \text { L }\end{array}$

958 ccatggacaaagattgatacacttgctttgtctttactgcgaaaagtgctgatgcctctg

$\begin{array}{llllllllllllllllllllll}241 & \text { P } & \text { W } & \text { T } & \text { K } & \text { I } & \text { D } & \text { T } & \text { L } & \text { A } & \text { L } & \text { S } & \text { L } & \text { L } & \text { R } & \text { K } & \text { V } & \text { L } & \text { M } & \text { P } & \text { L }\end{array}$

1018 ccagggaagcgttataccattcaacaaataactaaccatcagtggtttcaaaaaatattt

$\begin{array}{lllllllllllllllllllll}261 & P & G & K & R & Y & T & I & Q & Q & I & T & N & H & Q & W & F & Q & K & I & F\end{array}$

1078 aaagtttcaagtacatctcttagagcagaagaaaacacacctgtatctaaacggatatgt

$\begin{array}{lllllllllllllllllllll}281 & \mathrm{~K} & \mathrm{~V} & \mathrm{~S} & \mathrm{~S} & \mathrm{~T} & \mathrm{~S} & \mathrm{~L} & \mathrm{R} & \mathrm{A} & \mathrm{E} & \mathrm{E} & \mathrm{N} & \mathrm{T} & \mathrm{P} & \mathrm{V} & \mathrm{S} & \mathrm{K} & \mathrm{R} & \mathrm{I} & \mathrm{C}\end{array}$

1138 tctgacgccgtggacgecggtttgccaccgtcttcatcggacgccagccgcctgtcatac

$\begin{array}{llllllllllllllllllllll}301 & \text { S } & \text { D } & \text { A } & \text { V } & \text { D } & \text { A } & \text { G } & \text { L } & \text { P } & \text { P } & \text { S } & \text { S } & \text { S } & \text { D } & \text { A } & \text { S } & \text { R } & \text { L } & \text { S } & \text { Y }\end{array}$

1198 tctcagcctggcttgggtttcttctccggttcccaaccggtacaccaaaacgacaacaat

$\begin{array}{llllllllllllllllllllll}321 & \text { S } & Q & \text { P } & G & \text { L } & G & \text { F } & \text { F } & \text { S } & \text { G } & \text { S } & \text { Q } & \text { P } & \text { V } & \text { H } & \text { Q } & \text { N } & \text { D } & \text { N } & \text { N }\end{array}$

1258 gatgatgaagagccaataatcttccaggcgcagtgtttagcttctcccaaccegctcat

$\begin{array}{lllllllllllllllllllll}341 & \text { D } & \text { D } & \text { E } & \text { E } & \text { P } & \text { N } & \text { N } & \text { L } & \text { P } & \text { G } & \text { A } & \text { V } & \text { F } & \text { S } & \text { F } & \text { S } & \text { Q } & \text { P } & \text { A } & \text { H }\end{array}$

1318 attgacggtatgctacttaactcgcagttgaacacgcagaccgcttcaggttcaagcatg

$\begin{array}{lllllllllllllllllllll}361 & \text { I } & \text { D } & \text { G } & \text { M } & \text { L } & \text { L } & \text { N } & \text { S } & \text { Q } & \text { L } & \text { N } & \text { T } & \text { Q } & \text { T } & \text { A } & \text { S } & \text { G } & \text { S } & \text { S } & \text { M }\end{array}$

1378 agttctccactgcaaaggctcgttaaaaggatgactcgtttggtggctaaagtcagctgt

$\begin{array}{lllllllllllllllllllll}381 & \text { S } & \text { S } & \text { P } & \text { L } & \text { Q } & \text { R } & \text { L } & \text { V } & \text { K } & \text { R } & \text { M } & \text { T } & \text { R } & \text { L } & \text { V } & \text { A } & \text { K } & \text { V } & \text { S } & \text { C }\end{array}$

1438 gaagaagcaatcaagcatttgagccaacaactaatcaaacttggctacacttggaaaata

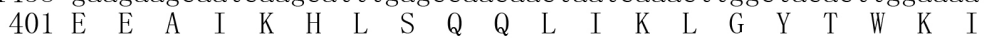

1498 cacacccccggagtggttactatatcaactcaagatcgacgcaaaatgcaacttgttttc

$\begin{array}{lllllllllllllllllllll}421 & \mathrm{H} & \mathrm{T} & \mathrm{P} & \mathrm{G} & \mathrm{V} & \mathrm{V} & \mathrm{T} & \mathrm{I} & \mathrm{S} & \mathrm{T} & \mathrm{Q} & \mathrm{D} & \mathrm{R} & \mathrm{R} & \mathrm{K} & \mathrm{M} & \mathrm{Q} & \mathrm{L} & \mathrm{V} & \mathrm{F}\end{array}$

1558 aaagcaacagtttatgatatgcagacaatggtgttgctcgactttcggctgtcgagagge

$\begin{array}{lllllllllllllllllllll}441 & \mathrm{~K} & \text { A } & \text { T } & \text { V } & \text { Y } & \text { D } & \text { M } & \text { Q } & \text { T } & \text { M } & \text { V } & \text { L } & \text { L } & \text { D } & \text { F } & \text { R } & \text { L } & \text { S } & \text { R } & \text { G }\end{array}$

1618 tgtggactggatttcaaagacatttcctagccattaaacataagttagctgatattttg

$\begin{array}{llllllllllllllllllllll}461 & \mathrm{C} & \mathrm{G} & \mathrm{L} & \mathrm{D} & \mathrm{F} & \mathrm{K} & \mathrm{R} & \mathrm{H} & \mathrm{F} & \mathrm{L} & \mathrm{A} & \mathrm{I} & \mathrm{K} & \mathrm{H} & \mathrm{K} & \mathrm{L} & \mathrm{A} & \mathrm{D} & \mathrm{I} & \mathrm{L}\end{array}$

1678 tgttcagcaccagtaacatggtcaatggccactgctactaatagcattcct tgalacaacc

$\begin{array}{lllllllllllllllllll}481 & \text { C } & \text { S } & \text { A } & \text { P } & V & T & W & \text { S } & \text { M } & \text { A } & \text { T } & \text { A } & \text { T } & \text { N } & \text { S } & \text { I } & \text { P } & *\end{array}$

1738 tttttaactatcttgttttataaatg ttgaattacacagagtgttttgtagcaaaaaaaa

1798 aaaaaaaaaaaaaaaaaaaa

Figure 1. Full-length DcarChk1 and corresponding amino acid sequence. Initiation (ATG) and termination (TGA) codon are highlighted in bold and boxed. S_TKc domain is shaded in yellow. 


\section{Analysis of DcarChk1 sequence and of the encoding protein structure}

DcarChk1 cDNA contained a 1494-bp ORF; 237-bp 5'-untranslated region (UTR); 86-bp 3'-UTR; and a 3'-polyA tail. The protein encoded by the ORF was 497 aa in length, and had a calculated MW of $55.84 \mathrm{kDa}$, a theoretical pl of 8.65, and no signal peptide. A catalytic domain with the activity of a serine/threonine-protein kinase (S_TKc domain) was detected at aa positions 21276 (Figure 1, shaded yellow).

\section{Analysis of DcarChk1 amino acid homology}

Amino acid sequences encoded by DcarChk1 were compared with those of other Chk1 homologs in GenBank (accession Nos. are given in Table 2). The highest level of homology was to Chk1 of D. pulex (94.37\%). Levels of homology with other species (Apis dorsata, Bombus terrestris, Megachile rotundata, Anopheles darlingi, Acyrthosiphon pisum, Metaseiulus occidentalis, Saccoglossus kowalevskii, Crassostrea gigas, Strongylocentrotus purpuratus, Ornithorhynchus anatinus, and Cavia porcellus) ranged from 50 to $54 \%$ (Figure 2). Upon phylogenetic tree construction, Chk1 homologs formed two divergent branches (Figure 3). D. carinata and D. pulex were closely related and clustered together with the hymenopteran insects and arachnids, while other species were more distantly related.

Table 2. Accession numbers of Chk1 from various species.
\begin{tabular}{l|l|l}
\hline Organism & Gene name (definition in NCBI) & Accession No \\
\hline Daphnia pulex & checkpoint kinase 1 & AGN95867 \\
\hline Apis dorsata & PREDICTED: serine/threonine-protein kinase grp-like & XP_006608254 \\
\hline Bombus terrestris & PREDICTED: serine/threonine-protein kinase grp-like & XP_003401057 \\
\hline Megachile rotundata & PREDICTED: serine/threonine-protein kinase grp-like & XP_003705992 \\
\hline Anopheles darlingi & Serine/threonine-protein kinase grp & ETN58880 \\
\hline Acyrthosiphon pisum & PREDICTED: serine/threonine-protein kinase grp-like & XP_001951665 \\
\hline Metaseiulus occidentalis & PREDICTED: serine/threonine-protein kinase Chk1-like & XP_003745318 \\
\hline Crassostrea gigas & Serine/threonine-protein kinase Chk1 & EKC39820 \\
\hline Saccoglossus kowalevskii & PREDICTED: serine/threonine-protein kinase Chk1-like & XP_002741282 \\
\hline Strongylocentrotus purpuratus & checkpoint kinase 1 & ABK80717 \\
\hline Danio rerio & PREDICTED: serine/threonine-protein kinase Chk1 isoform X1 & XP_005161214 \\
\hline Anolis carolinensis & PREDICTED: serine/threonine-protein kinase Chk1-like & XP_003225759 \\
\hline Pseudopodoces humilis & PREDICTED: serine/threonine-protein kinase Chk1 & XP_005528500 \\
\hline Cavia porcellus & PREDICTED: serine/threonine-protein kinase Chk1 & XP_003472613 \\
\hline Ornithorhynchus anatinus & PREDICTED: serine/threonine-protein kinase Chk1 & XP_001513209 \\
\hline
\end{tabular}

\section{PCR analysis of DcarChk1 expression in different reproductive phases}

DcarChk1 expression was detected in both females and males. Levels of expression were significantly higher in males than in females $(P<0.01)$, and in sexual females than in parthenogenetic females (Figure 4). Expression of DcarChk1 was also found to be higher in adults than in larvae, regardless of the sexual phase of the females (Figure 4).

\section{Cellular localization of DcarChk1 mRNA expression in parthenogenetic and sexual females}

DcarChk1 mRNA expression and cellular localization in different reproductive phases of Daphnia females were investigated using a digoxin-labeled RNA riboprobe, which bound to cell- 

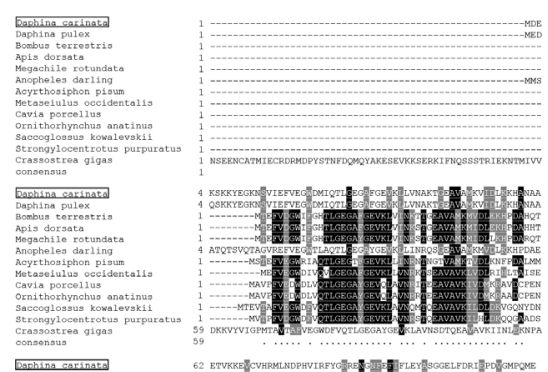

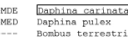
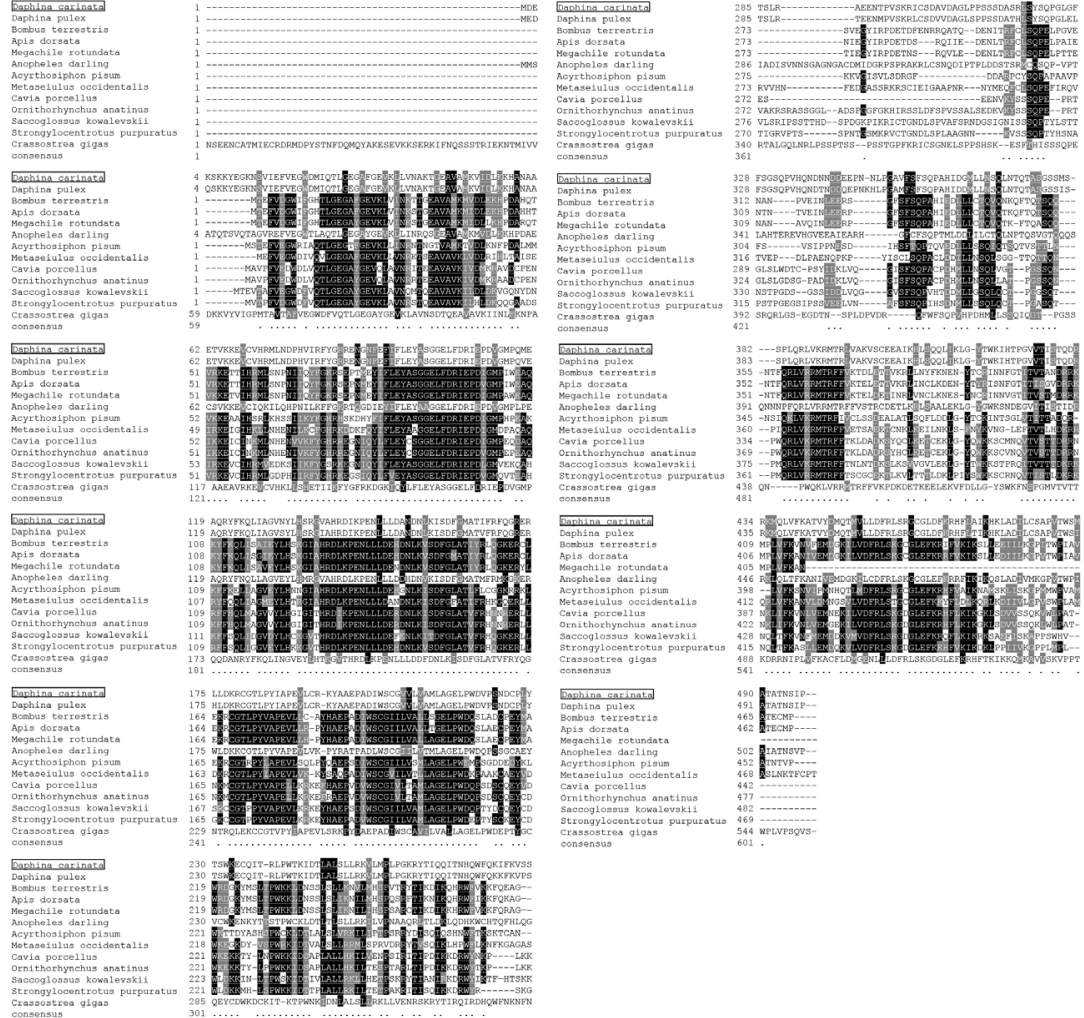

Figure 2. Multiple amino acid sequence alignment of DcarChk1 to its closest homologs. Identical (black) and similar (gray) amino acids are shown.

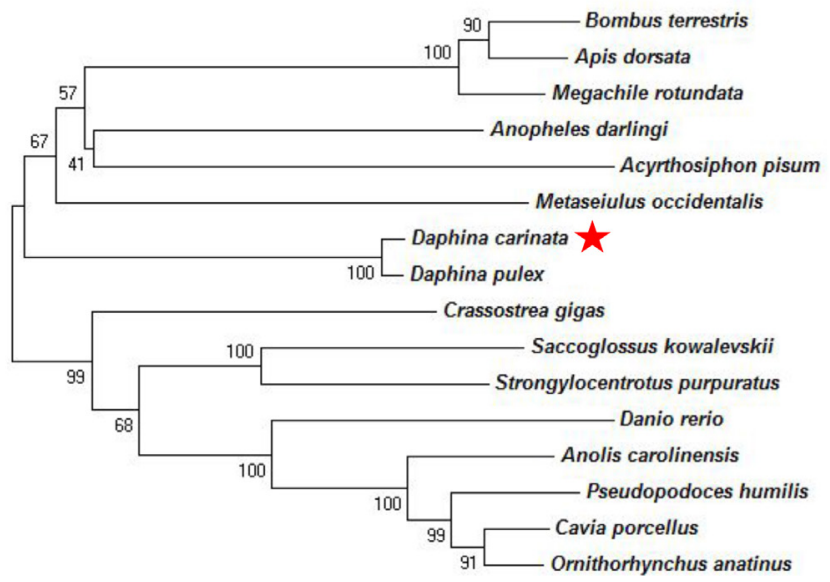

$\longmapsto 0.05$

Figure 3. Neighbor-joining tree showing relationship of DcarChk1 with homologs from other species. 
specific mRNA. DcarChk1 mRNA in parthenogenetic females was expressed mainly on the head surface, capillus, and carapace valve edge (Figure $5 \mathrm{~A}$ and $\mathrm{C}$ ). In sexual females, expression was concentrated mainly in the joint of the second antennae and on the thoracic limbs and capillus (Figure 5B and D).

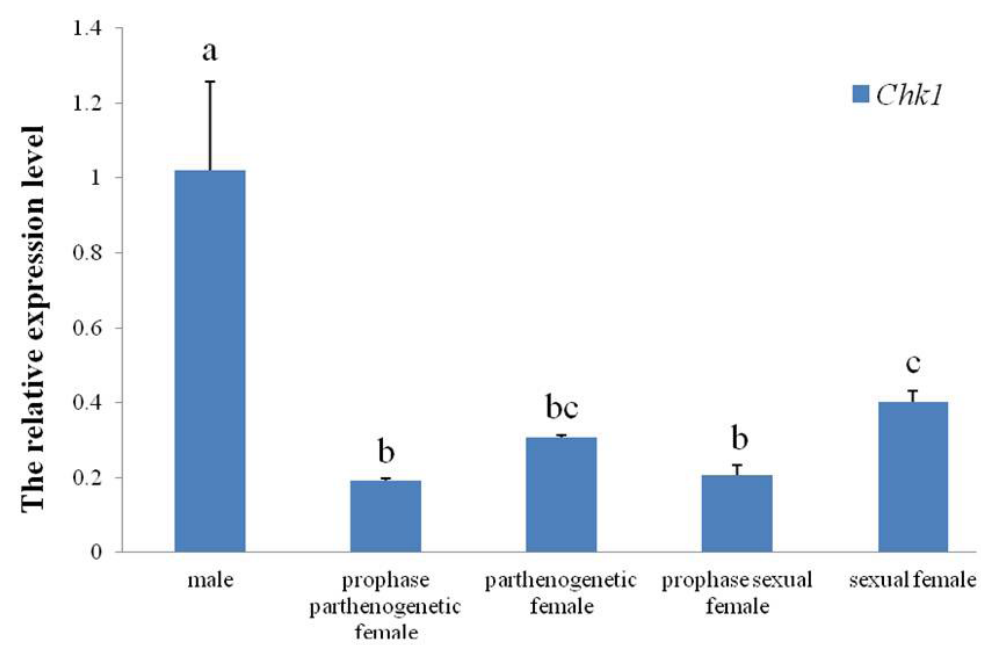

Figure 4. Expression of DcarChk1 in Daphnia carinata males and females at different reproductive phases by real-time qPCR analysis. Labeled bars $(a, b, c)$ indicate significant differences $(P<0.05)$.

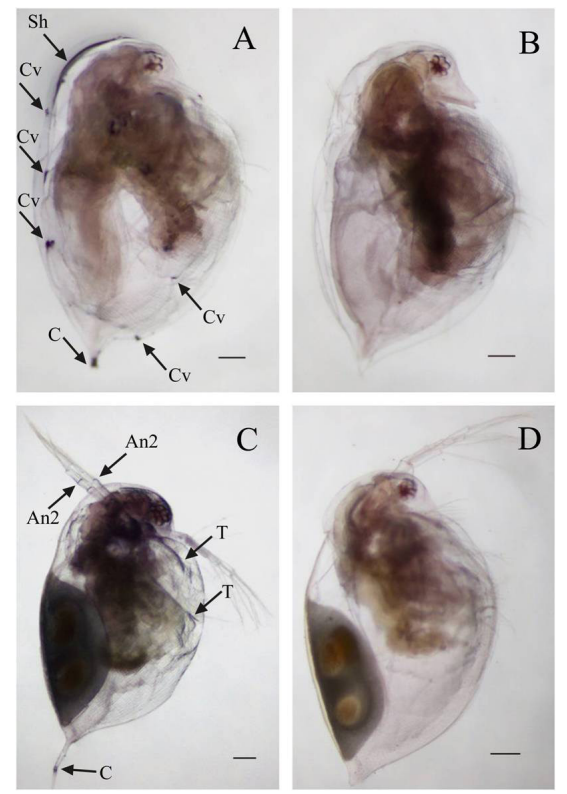

Figure 5. DcarChk1 in parthenogenetic and sexual Daphnia carinata females. Localization by in situ hybridization. (A. C. DIC-labeled DcarChk1 antisense probe; B. D. DIC-labeled DcarChk1 sense probe). Blue-violet area shows positive signals. Sh: surface of head; C: capillus; Cv: carapace valve; An2: second antenna; T: thoracic limbs. Scale bars indicate $200 \mu \mathrm{m}$. 


\section{DISCUSSION}

Living organisms possess a complex cell cycle checkpoint signaling pathway, in which DNA damage triggers a series of cascading reactions (Wang et al., 2005). As an effector kinase, the stable expression of Chk1, and its protein, aids in repairing damaged DNA and regulating this cycle, thereby ensuring the integrity and stability of the cellular genome (Wang et al., 2006b). Chk1 encodes a type of serine/threonine-protein kinase belonging to the protein kinase family, within the tyrosine-protein kinases. Protein kinase functionality is evolutionarily conserved across lineages (Manning et al., 2002a) due to the involvement of these proteins in a multitude of cellular processes including division, proliferation, apoptosis, and differentiation (Manning et al., 2002b). Protein kinases catalyze the transfer of gamma phosphate from ATP to one or more aa residues in a protein substrate side-chain, resulting in phosphorylation of substrate proteins. Phosphorylation usually results in a conformational change of the target protein - by changing enzyme activity, cellular location, or associations with other proteins - which affects its function (Hanks et al., 1988). In the current study, we observed that the encoding protein of DcarChk1 contained a domain with catalytic activity typical of serine/threonine-protein kinases, suggesting that the encoding protein of DcarChk1 is indeed a kind of serine/threonine-protein kinase; this in turn indicates that DcarChk1 plays an important role in the growth and development of $D$. carinata.

Protein aa sequence encoded by DcarChk1 of $D$. carinata had the highest homology with another crustacean species, D. pulex, followed by members of the order Insecta. This finding supports the "Pancrustacea" theory, that insects and crustaceans share a common ancestor (Yin, 2003; Song, 2006).

Expression of DcarChk1 was higher in males than in females; higher in sexual females than parthenogenetic females; and higher in adults than in larvae regardless of the sexual phase of females. The protein encoded by DcarChk1 is thought to function as a negative regulator of cell division (Pabla et al., 2012). During the early development of $D$. carinata (the larval stage), when cellular division is rapid, lower levels of DcarChk1 expression were detected than levels observed in adults. This finding suggests that DcarChk1 plays an important role in the growth and development of this species. The appearance of sexual females and males in this species occurs in response to worsening environmental conditions. Deteriorating habitats can damage DNA, increasing the probability of gene mutation caused by base mismatch. High levels of expression of DcarChk1 in sexual females and males would ensure timely repair of damaged DNA, and the maintenance of a normal cell cycle. The observed difference in expression levels of DcarChk1 between males and females, as well as between females in different sexual phases, indicates that expression is sex-dependent and suggests that DcarChk1 is involved in controlling reproductive transformation of $D$. carinata. Specific underlying mechanisms for this control, however, are yet to be determined.

In situ hybridization has recently emerged as an important means of gene mapping (Gall and Pardue, 1969; Thisse and Thisse, 2008; Zhang et al., 2007; Zhang and Liu, 2013). Using this technique in the current study, we observed that DcarChk1 was expressed in parthenogenetic females mainly on the head surface, capillus, and carapace valve edge. In sexual females, expression was seen mainly in the joint of the second antennae and on the thoracic limbs and capillus. The carapace valve of cladocerans is similar to that of other crustaceans and is formed from protrusions extending from the rear of the head and the cuticle produced by the outer layer of the epithelium folds. The carapace of Daphnia, which is thin, soft and transparent, functions to protect the body and thoracic limbs and can also serve to harbor hatchlings (Jiang and Du, 1979). 
During growth, the carapace valve is shed as individuals molt. Chk1, as an effector kinase, may act to maintain smooth functioning of the cell cycle during this time. Hence, the high expression of DcarChk1 in the carapace valve observed in our study may indicate that this gene has an important function in the timely molt of $D$. carinata.

The capillus of $D$. carinata has a role in immune defense and is formed by the extension of the postero-dorsal and -ventral angles of the carapace valve (Zhang et al., 2009). The second antennas, which are stronger than the first antennas, grow on both sides of the head and aid in swimming locomotion (Jiang and Du, 1979). Expression of DcarChk1 in these areas may contribute to the maintenance of normal physical activity. Unlike other crustaceans, the thoracic limbs of cladocerans are modified and immobile, and function as feeding and respiratory organs (Jiang and Du, 1979). Additionally, the thoracic limbs are used during mating and oogenesis and involved in a dimorphic characteristic between males and females. In males, the endopodite of the first thoracic limb forms a hamulus, and the exopodite develops a long flagellum, which functions to grasp the female during mating (Lin et al., 2008). Gene mapping showing expression of DcarChk1 in the thoracic limbs of $D$. carinata indicates that this gene has a role in sexual reversal between males and females. Results from the present study lend further support to the importance of DcarChk1 in the growth and development of $D$. carinata. Further study is required to more robustly understand specific mechanisms of function of DcarChk1 in this species.

\section{ACKNOWLEDGMENTS}

Research supported by the National Natural Science Foundation of China (grant \#31172043); the Natural Science Foundation of Zhejiang, China (grant \#LY12C19003); and the Priority Discipline Open Fund Projects of Zhejiang, China (grant \#xkzsc1409). The authors are grateful to the College of Life Sciences of Ningbo University and the Shanghai Center for Plant Stress Biology Chinese Academy of Sciences for the provision of technical support. Elements of the study were conducted in the Instruments Sharing Platform of School of Life Sciences, East China Normal University (ECNU).

\section{REFERENCES}

Bartek J and Lukas J (2003). Chk1 and Chk2 kinases in checkpoint control and cancer. Cancer Cell 3: 421-429. http://dx.doi. org/10.1016/S1535-6108(03)00110-7

Bolderson E, Richard DJ, Zhou BB and Khanna KK (2009). Recent advances in cancer therapy targeting proteins involved in DNA double-strand break repair. Clin. Cancer Res. 15: 6314-6320. http://dx.doi.org/10.1158/1078-0432.CCR-09-0096

Cao WQ, Lin YS and Guo DH (2001). Effect studies of some environmental conditions on reproductive conversion of Moina mongolica, a salt water cladocera. J. Oceanogr. Taiwan 20: 190-195.

Carrassa L, Sanchez Y, Erba E and Damia G (2009). U2OS cells lacking Chk1 undergo aberrant mitosis and fail to activate the spindle checkpoint. J. Cell. Mol. Med. 13 (8A): 1565-1576. http://dx.doi.org/10.1111/j.1582-4934.2008.00362.x

Chen CC, Kennedy RD, Sidi S, Look AT, et al. (2009). CHK1 inhibition as a strategy for targeting Fanconi Anemia (FA) DNA repair pathway deficient tumors. Mol. Cancer 8: 24. http://dx.doi.org/10.1186/1476-4598-8-24

Chen Y and Sanchez Y (2004). Chk1 in the DNA damage response: conserved roles from yeasts to mammals. DNA Repair (Amst.) 3: 1025-1032. http://dx.doi.org/10.1016/j.dnarep.2004.03.003

Gall JG and Pardue ML (1969). Formation and detection of RNA-DNA hybrid molecules in cytological preparations. Proc. Natl. Acad. Sci. USA 63: 378-383. http://dx.doi.org/10.1073/pnas.63.2.378

Hanks SK, Quinn AM and Hunter T (1988). The protein kinase family: conserved features and deduced phylogeny of the catalytic domains. Science 241: 42-52. http://dx.doi.org/10.1126/science.3291115

Hebert PDN (1978). The population biology of Daphnia (Crustacea, Daphnidae). Biol. Res. 53: 387-426. http://dx.doi. 
org/10.1111/j.1469-185X.1978.tb00860.x

Jiang XZ and Du NS (1979). Fauna Sinica, Crustacea, Freshwater Cladocera. Beijing: Science Press, 8-15.

Khmeleva NN, Golubev AP and Kidava A (1995). Growth and reproduction of Daphnia magna under changing photoperiod and temperature conditions. Doklady Akademii nauk BSSR 39: 71-74.

Li CJ, Liu KY, Yang DY, Yu ZH, et al. (2006). Progress in Checkpoint Kinase 1. Chin. J. Cell Biol. 28: 149-152.

Lin L, Zhang MF, Zhao YL, Guo ZL, et al. (2008). External morphological study on the male Daphnia carinata. J. Fudan Univ. Nat. Sci. 47: 354-358.

Ling H, Wen L, Ji XX, Tang YL, et al. (2010). Growth inhibitory effect and Chk1-dependent signaling involved in G2/M arrest on human gastric cancer cells induced by diallyl disulfide. Braz. J. Med. Biol. Res. 43: 271-278. http://dx.doi.org/10.1590/ S0100-879X2010007500004

Liu DF, Li B, Jiang H, Ma LL, et al. (2010). [Expression of Chk1 in human sperm and its significance]. Zhonghua Nan Ke Xue 16: 811-815.

Livak KJ and Schmittgen TD (2001). Analysis of relative gene expression data using real-time quantitative PCR and the 2(-Delta Delta C(T)) Method. Methods 25: 402-408. http://dx.doi.org/10.1006/meth.2001.1262

Manning G, Plowman GD, Hunter T and Sudarsanam S (2002a). Evolution of protein kinase signaling from yeast to man. Trends Biochem. Sci. 27: 514-520. http://dx.doi.org/10.1016/S0968-0004(02)02179-5

Manning G, Whyte DB, Martinez R, Hunter T, et al. (2002b). The protein kinase complement of the human genome. Science 298: 1912-1934. http://dx.doi.org/10.1126/science.1075762

Martinez-Jeronimo F, Villasenor R, Rios G and Espinosa F (1994). Effect of food type and concentration on the survival, longevity and reproduction of Daphnia magna. Hydrobiologia 287: 207-214. http://dx.doi.org/10.1007/BF00010735

Merry C, Fu K, Wang J, Yeh IJ, et al. (2010). Targeting the checkpoint kinase Chk1 in cancer therapy. Cell Cycle 9: 279-283. http://dx.doi.org/10.4161/cc.9.2.10445

Pabla N, Bhatt K and Dong Z (2012). Checkpoint kinase 1 (Chk1)-short is a splice variant and endogenous inhibitor of Chk1 that regulates cell cycle and DNA damage checkpoints. Proc. Natl. Acad. Sci. USA 109: 197-202. http://dx.doi.org/10.1073/ pnas. 1104767109

Pan Y, Ren KH, He HW and Shao RG (2009). Knockdown of Chk1 sensitizes human colon carcinoma HCT116 cells in a p53dependent manner to lidamycin through abrogation of a G2/M checkpoint and induction of apoptosis. Cancer Biol. Ther. 8: 1559-1566. http://dx.doi.org/10.4161/cbt.8.16.8955

Qi S, Wu MG and Zhang X (1991). The biological characteristics of three cladocerans and acute toxic test. J. Jinan Univ. 12: 73-78.

Rouse J and Jackson SP (2002). Interfaces between the detection, signaling, and repair of DNA damage. Science 297: 547551. http://dx.doi.org/10.1126/science. 1074740

Slupianek A, Hoser G, Majsterek I, Bronisz A, et al. (2002). Fusion tyrosine kinases induce drug resistance by stimulation of homology-dependent recombination repair, prolongation of $\mathrm{G}(2) / \mathrm{M}$ phase, and protection from apoptosis. Mol. Cell. Biol. 22: 4189-4201. http://dx.doi.org/10.1128/MCB.22.12.4189-4201.2002

Song DX (2006). Classification and evolution of arthropods. Bull. Biol. 41: 1-3.

Sørensen CS, Hansen LT, Dziegielewski J, Syljuåsen RG, et al. (2005). The cell-cycle checkpoint kinase Chk1 is required for mammalian homologous recombination repair. Nat. Cell Biol. 7: 195-201. http://dx.doi.org/10.1038/ncb1212

Stross RG and Hill JC (1965). Diapause induction in Daphnia requires two stimuli. Science 150: 1462-1464. http://dx.doi. org/10.1126/science.150.3702.1462

Syljuåsen RG, Sørensen CS, Hansen LT, Fugger K, et al. (2005). Inhibition of human Chk1 causes increased initiation of DNA replication, phosphorylation of ATR targets, and DNA breakage. Mol. Cell. Biol. 25: 3553-3562. http://dx.doi.org/10.1128/ MCB.25.9.3553-3562.2005

Thisse C and Thisse B (2008). High-resolution in situ hybridization to whole-mount zebrafish embryos. Nat. Protoc. 3: 59-69. http://dx.doi.org/10.1038/nprot.2007.514

Walworth N, Davey S and Beach D (1993). Fission yeast chk1 protein kinase links the rad checkpoint pathway to cdc2. Nature 363: 368-371. http://dx.doi.org/10.1038/363368a0

Wang HY, Zhang M and Zou P (2005). Advances in the research of checkpoint kinase 1. J. Med. Mol. Biol. 2: 147-149.

Wang HY, Zhang M, Zou P, You Y, et al. (2006a). [Mechanism of G2/M blockage triggered by activated-Chk1 in regulation of drug-resistance in K562/A02 cell line]. Zhongguo Shi Yan Xue Ye Xue Za Zhi 14: 1105-1109.

Wang YX, Zhu SC, Wang X, Feng W, et al. (2006b). Expression of CHK1 and CHK2 protein in tissue of esophageal carcinoma. Cancer Res. Prevent. Treat. 33: 171-174.

Yang SM (1994). Fishes feeding promoting matter and advanced fish farming. J. Fish. China 18: 80-83.

Yin WY (2003). Introduction to a new hypothesis "Pancrustacea": a review of the progress of arthropod phylogeny. Zool. Res. 24: 11-16. 
Zhang CX and Liu F (2013). [A brief protocol for high-resolution whole mount in situ hybridization in zebrafish]. Yi Chuan 35 : 522-528. http://dx.doi.org/10.3724/SP.J.1005.2013.00522

Zhang M, Wang HY, You Y, Chen ZC, et al. (2008). Inhibition of HeLa cell proliferation and induction of apoptosis by CHK1 shRNA. Chin. J. Pathophysiol. 24: 1891-1894.

Zhang MF, Zeng C, Chen YS and Zhao YL (2009). Karyologic observation on maturation of germ cells of Daphnia (Ctenodaphnia) carinata. J. Fish. Sci. China 16: 868-877.

Zhang RF, Hou L, Zou XY, Zhao XT, et al. (2007). A study on the related genes of early embryonic development of Artemia sinica - establishment of whole-mount fluorescent in situ hybridization to Artemia sinica Nauplius' Trachealess gene. Trans. Oceanol. Limnol. 29: 88-92. 